\title{
The ion beam shepherd: A new concept for asteroid deflection
}

\author{
Claudio Bombardelli*, Hodei Urrutxua, Mario Merino, Jesús Peláez, Eduardo Ahedo \\ School of Aeronautical Engineering, Technical University of Madrid, Plaza Cardenal Cisneros, 3, 28040 Madrid, Spain
}

\begin{abstract}
A novel slow push asteroid deflection strategy has been recently proposed in which an Earth threatening asteroid can be deflected by exploiting the momentum transmitted by a collimated beam of quasi-neutral plasma impinging against the asteroid surface. The beam can be generated with state-of-the art ion engines from a hovering spacecraft with no need for physical attachment or gravitational interaction with the celestial body. The spacecraft, placed at a distance of a few asteroid diameters, would need an ion thruster pointed at the asteroid surface as well as a second propulsion system to compensate for the ion engine reaction and keep the distance between the asteroid and the shepherd satellite constant throughout the deflection phase. A comparison in terms of required spacecraft mass per total imparted deflection impulse shows that the method outperforms the gravity tractor concept by more than one order of magnitude for asteroids up to about $200 \mathrm{~m}$ diameter. The two methods would yield comparable performance for asteroids larger than about $2 \mathrm{~km}$.
\end{abstract}

\section{Introduction}

The deflection of an asteroid from its collision course with our planet is, in principle, technically feasible and can be carried out in different ways. The basic idea is to transmit a deflecting impulse to the asteroid whose effect is to eventually produce a large enough shift in the asteroid intersection point on the Earth $b$-plane in such a way that the impact with our planet can be ruled out with a high enough degree of confidence.

Such impulse can be transmitted instantaneously by use of a stand-off nuclear explosion or simply by having a spacecraft impacting against it at high relative velocity [1-3]. The latter method, often referred to as kinetic impactor $(\mathrm{KI})$, is one of the preferred asteroid deflection methods, mostly due to the fact that the technique has been successfully demonstrated by the NASA Deep Impact mission in 2005. In that mission, a $370-\mathrm{kg}$ impactor collided at $10.3 \mathrm{~km} / \mathrm{s}$ relative velocity with the nucleus of the P/Tempel 1 comet. Nevertheless, impacting at a similar speed against a typical $150 \mathrm{~m}$ diameter asteroid, roughly 40 times smaller than the above comet, would require advances in the field of guidance and navigation not to be underestimated.

A radically different strategy consists of building up the required deflection impulse with a long-duration slow push transmitted to the asteroid by a rendezvous spacecraft. The key advantage of this solution is that there is no risk of fracturing the target asteroid, which may be fragile especially when in a fast rotational state [4]. In addition, unlike the impact methods, the deflection can be carried out in a precise manner and its outcome can be tracked and accurately predicted.

Among the "slow-push" methods the gravity tractor (GT) concept, proposed by Lu and Love in 2005 [5], is regarded as one of the most promising. When using a GT the deflection can be achieved without physical contact between the spacecraft and the asteroid and can be predicted very accurately once the distance and the mass 
of the asteroid and the spacecraft are known, irrespective of the asteroid surface and structural properties.

However, the gravity tractor concept suffers from at least two major drawbacks [6]. The first is the need for a massive spacecraft to physically produce the gravitational force required to slowly deflect the asteroid. The second is the difficulty of controlling the (unstable) proximity hovering position of the spacecraft for a large period of time avoiding undesired collisions [5].

The recently proposed ion beam shepherd concept (IBS) [6] promises to overcome these limitations by using the momentum of impinging propellant ions rather than gravity to achieve a contactless slow-push deflection, whose magnitude does not depend on the spacecraft and asteroid mass but rather on the characteristics of the power and propulsion system employed. Ion thrusters tested in previous space missions can be used for this purpose without the need of substantial modifications, although an improvement in terms of specific impulse and beam divergence would increase the IBS capability. Reducing the beam divergence, i.e. the half-angle of the conical envelope containing the quasi-totality of the beam current (typically a 95\% value is employed in the definition of divergence) would prove to be particularly effective as it would allow larger hovering distances without having a significant fraction of the beam missing the asteroid.

Another point of strength of the method is that the ionic propulsion system can be used for both the interplanetary low-thrust trajectory and the deflection manoeuvre. In other words, the deflection system is part of the spacecraft system.

The key aspects of the concept, which has been also proposed for active space debris removal [7], are summarised in this article where an extensive comparison with the gravity tractor concept is also performed.

\section{Concept description}

The IBS concept is schematized in Fig. 1. The shepherd spacecraft is located not too far from the asteroid, pointing one of its ion thrusters directly at the asteroid surface. The high-velocity ions of the quasi-neutral plasma emitted by the thruster reach the asteroid surface, penetrating the asteroid material while losing their energy through ionising collisions until they stop, typically a few nanometres below the surface. If the beam fully intercepts the asteroid the latter will undergo a force roughly equal and opposite to the one experienced by the spacecraft. It will then be necessary to have a second ion thruster mounted on the spacecraft to cancel out the total force and keep constant the distance with respect to the asteroid. Note that the IBS gravitational pull on the asteroid, which would, in principle, reduce the transmitted momentum is typically negligible for reasonable hovering distances as shown in [6] and later on in this article.

\section{IBS mass cost}

When the deflection starts a few years before the impact event, as it is typically the case for slow-push methods, and no intermediate planetary flybys occur, the optimum push strategy consists of changing the asteroid semimajor axis in such a way that it will arrive at the predicted impact location with an accumulated time delay. This, in turn, produces a shift along the $\zeta$ axis of the $b$-plane, as defined by Valsecchi et al. [8], which almost coincides with the total achieved deflection (the shift along the $\xi$ axis, which corresponds to the minimum orbit intersection distance or MOID, is usually negligible). In order to produce the maximum variation of semimajor axis over a finite time interval the thrust direction has to be aligned with the primer vector of the Hamiltonian constructed around the final orbital energy to be maximised. For extremely small accelerations, as is the case here, such vector is virtually coincident with the tangent to the orbit at all times.

If for simplicity we assume the applied force $F$ is constant and the primary and secondary propulsion systems are identical, the total mass $m_{\text {tot }}$ needed for such a slow-push campaign of duration $\Delta t$ can be divided into fuel, power plant and structure:

$m_{I B S}=2 F\left[\frac{\Delta t}{I_{s p} g}+\frac{\alpha I_{s p} g}{2 \eta_{e p}}\right]+m_{s t r}$

where $I_{s p}$ is the (constant) thruster specific impulse, $g$ is the sea-level gravity, $\eta_{e p}$ the thruster efficiency, $m_{s t r}$ the spacecraft structural mass (excluding the power plant) and $\alpha$ the inverse specific power, also considered as a constant (for simplicity).

Depending on the total thrust time $\Delta t$, the inverse specific power and the thruster efficiency there exists an optimum value of the specific impulse minimising the total spacecraft mass

$I_{s p}^{*}=\frac{1}{g} \sqrt{\frac{2 \eta_{e p} \Delta t}{\alpha}}$

which substituted in Eq. (1) provides the minimum mass required by the IBS system at the beginning of the

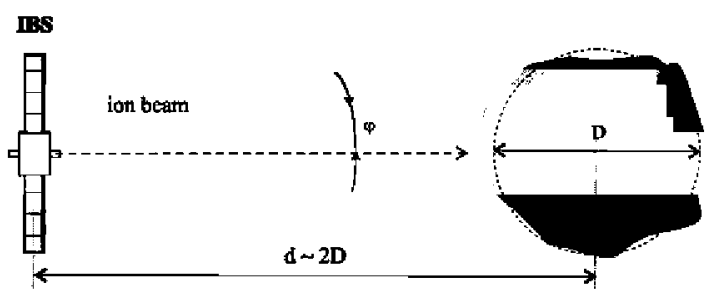

Fig. 1. Schematic of asteroid deflection with an ion beam shepherd 

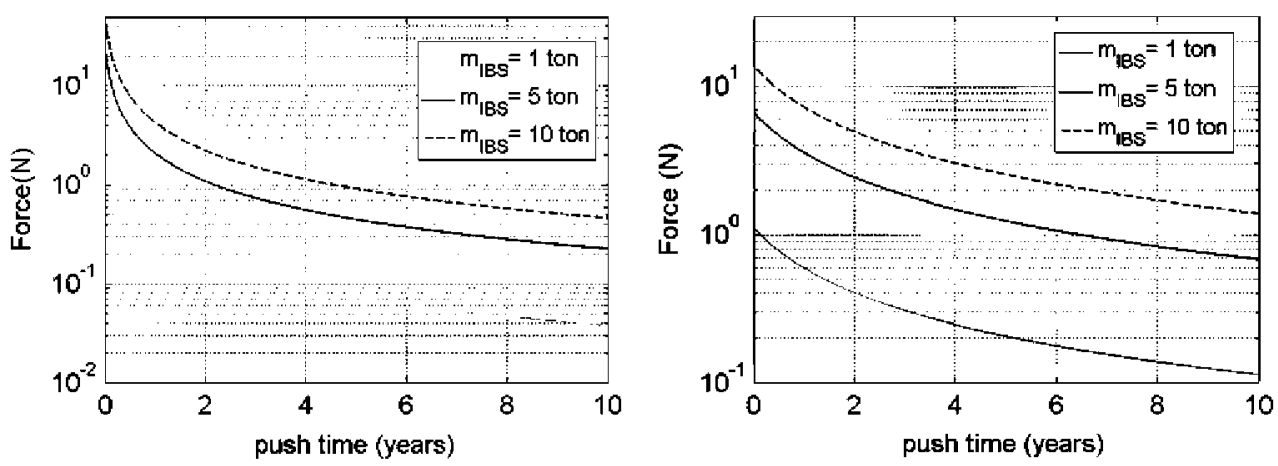

Fig. 2. Deflection force achievable as a function of the push time for different IBS masses and considering a specific impulse of $3000 \mathrm{~s}$ (left) and $10,000 \mathrm{~s}$ (right). Thruster efficiency is set to $70 \%$, inverse specific power to $5 \mathrm{~kg} / \mathrm{kW}$, and structural mass to $150 \mathrm{~kg}$.

deflection manoeuvre:

$m_{I B S}^{*}=2 F \sqrt{\frac{2 \alpha \Delta t}{\eta_{e p}}}+m_{s t r}$

From Eq. (2) one can see that for a typical thrusting time of 2 years and assuming $\alpha=10 \mathrm{~kW} / \mathrm{kg}$ held constant throughout the mission (for simplicity) and 70\% thrust efficiency an optimum specific impulse of $9600 \mathrm{~s}$ would be required. A specific impulse of almost 20,000s has been achieved in a plasma chamber [9], although space qualified ion thrusters can currently offer up to about 3000 s specific impulse.

Fig. 2 plots the achievable deflection force for an IBS system as a function of the desired push time and for two different values of the specific impulse.

\section{Gravity tractor comparison}

For asteroid deflection missions, characterised by a very large value of the total thrust impulse $F \Delta t$, a simple figure of merit for comparing different deflecting methods is the ratio of such impulse to the total spacecraft mass needed to obtain it, that is the total deflection impulse over spacecraft mass $(\mathrm{m} / \mathrm{s})$ :

$\chi=\frac{F \Delta t}{m_{S C}}$

From Eq. (3) and neglecting the $S / C$ structural mass the corresponding value for an IBS spacecraft yields

$\chi_{I B S} \approx \eta_{B} \sqrt{\frac{\eta_{e p} \Delta t}{8 \alpha}}$

where the beam momentum transfer efficiency, $0<\eta_{B}<1$, is added to account for the possibility that a fraction of the ion beam current misses the asteroid surface.

The mass of a gravity tractor providing a pull force $F$ from a hovering distance $d_{h o v}$ measured from the centre of a spherical asteroid of diameter $D_{a s t}$ and uniform material density $\rho_{\text {ast }}$ is

$m_{G T}=\frac{6 F d_{\mathrm{hov}}^{2}}{\pi G \rho_{a s t} D_{a s t}{ }^{3}}$
Such mass can be reduced by reducing the hovering distance $d_{h o v}$. Assuming a minimum safe hovering distance of 1.5 asteroid radii [3] one obtains the minimum required gravity tractor mass providing a pull force $F$ :

$m_{G T}^{*}=\frac{27 F}{8 \pi G \rho_{a s t} D_{a s t}}$

The corresponding total deflection impulse over spacecraft mass yields

$\chi_{G T}=\frac{8 \pi}{27} G \rho_{a s t} D_{a s t} \Delta t$

It is important to remark that the above value may not be achievable for very large asteroids. There is in fact a limit beyond which the propulsion and power system mass required to counteract the asteroid gravity exceed the value $m_{G T}^{*}$ specified by Eq. (7). In such a case the propulsion system capability will reach the saturation limit and the tractor hovering distance will need to be increased.

If we assume a thruster canting angle of $60^{\circ}$ [5], a hovering distance of one and a half asteroid radii from the centre [5] and negligible structural mass the asteroid diameter leading to the saturation of the propulsion system can be obtained by equating the equilibrium mass provided by Eq. (7) with the total power and propellant mass of the spacecraft. For an optimised specific impulse (Eq. (2)) one has

$2 F \sqrt{\frac{2 \alpha \Delta t}{\eta_{e p}}}=\frac{27 F}{8 \pi G \rho_{a s t} D_{w e t}^{*}}$

leading to the "saturation" diameter

$D_{a s t}^{*}=\frac{27 \sqrt{\eta_{e p}}}{16 \pi G \rho_{a s t} \sqrt{2 \alpha \Delta t}}$

For a typical asteroid material density $\rho_{a s t}=2 \mathrm{~kg} / \mathrm{cm}^{3}$, a thrust time of 2 years, a propulsion efficiency of $70 \%$ and an inverse power density $\alpha=5 \mathrm{~kW} / \mathrm{kg}$ we obtain $D_{\text {ast }}^{*} \approx 4 \mathrm{~km}$. Taking $\alpha=20 \mathrm{~kW} / \mathrm{kg}$ would provide $D_{a s t}^{*} \approx 2 \mathrm{~km}$.

Fig. 3 plots the ratio $\chi_{I B S} / \chi_{G T}$, derived from Eqs. (5) and (8) as a function of the diameter of an homogeneous spherical asteroid of mass density $\rho_{\text {ast }}=2 \mathrm{~kg} / \mathrm{cm}^{3}$. A thruster efficiency $\eta_{e p}=0.7$, a beam momentum transfer efficiency $\eta_{B}=0.9$ and a thrust duration of 2 years have been assumed. Two values of the inverse power density of 
$5 \mathrm{~kW} / \mathrm{kg}$ and $20 \mathrm{~kW} / \mathrm{kg}$ were considered to account for high efficiency solar array and onboard nuclear power generation systems, respectively.

As the asteroid diameter becomes smaller the difference in performance between the two methods rapidly increases with an order of magnitude difference for asteroids smaller than $200 \mathrm{~m}(400 \mathrm{~m})$ considering a power density of $20 \mathrm{~kg} / \mathrm{kW}(5 \mathrm{~kg} / \mathrm{kW})$. For very small asteroids $\left(20 \mathrm{~m}<D_{a s t}<40 \mathrm{~m}\right)$ a two-order-of-magnitude difference in performance is obtained.

For diameter larger than $2-4 \mathrm{~km}$ a saturation of the gravity tractor propulsion system is reached and the two methods would yield comparable performance.

Before analysing the IBS deflection performance for real asteroids we take advantage of the calculations performed

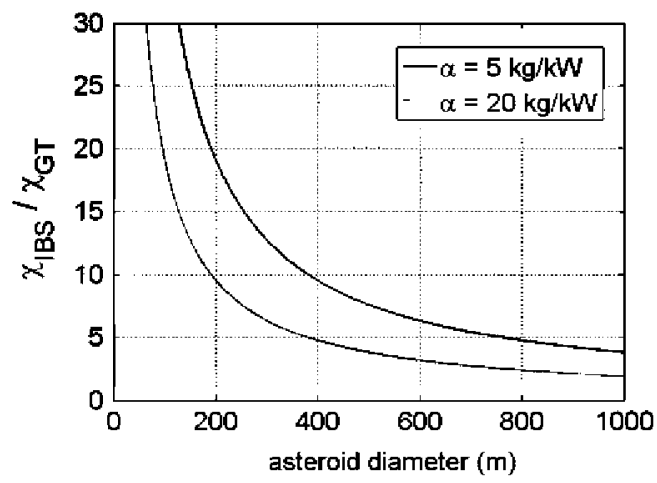

Fig. 3. Ratio of the two total deflection impulses per $\mathrm{kg}$ of spacecraft mass corresponding to the ion beam shepherd (IBS) and gravity tractor concept (GT), for two values of the inverse specific power generated on board.

Table 1

Main characteristics of the two chosen asteroids.

\begin{tabular}{lll}
\hline & $\mathbf{2 0 0 7} \mathbf{V K}_{\mathbf{1 8 4}}$ & $\mathbf{2 0 1 1} \mathbf{A G}_{\mathbf{5}}$ \\
\hline Mass (kg) & $3.3 \times 10^{9}$ & $3.9 \times 10^{9}$ \\
Diameter (m) & 130 & 140 \\
Semimajor axis (AU) & 1.726 & 1.43 \\
Eccentricity & 0.57 & 0.39 \\
Inclination (deg.) & 1.22 & 3.68 \\
\hline
\end{tabular}

above to express the ratio of the ion beam force transmitted to the asteroid to the gravitational pull on the shepherd spacecraft. By use of Eq. (3), neglecting the structure mass, and Eq. (6) we obtain

$\frac{F}{F_{\mathrm{g}}}=\frac{3 \eta_{B} \delta_{\text {hov }}^{2}}{2 \pi G \rho_{a s t} D_{a s t}} \sqrt{\frac{2 \eta_{e p}}{\alpha \Delta t}}$

where $\delta_{\text {hov }}$ is the hovering distance from the asteroid centre measured in asteroid diameters. After setting $\delta_{h o v}=2$, $\rho_{\text {ast }}=2 \mathrm{~kg} / \mathrm{cm}^{3}, \eta_{e p}=0.7, \eta_{B}=0.9, \Delta t=2$ years, and $\alpha=5$ $20 \mathrm{~kW} / \mathrm{kg}$, one can see that the gravitational pull is more than one order of magnitude smaller than the force transmitted to the asteroid for asteroid diameters below $2 \mathrm{~km}$. A two orders of magnitude difference appears for asteroids smaller than about $300 \mathrm{~m}$.

\section{Deflection performance}

In order to assess the achievable deflection in a real mission scenario we have considered the asteroids 2007 $\mathrm{VK}_{184}$ and $2011 \mathrm{AG}_{5}$ Table 1, currently the only two NEOs having index 1 with respect to the Torino scale, and applied a continuous tangential thrust acceleration throughout a given time interval before the impact. The orbital elements of the two asteroids, of $130 \mathrm{~m}$ and $140 \mathrm{~m}$ diameter, correspondingly, have been slightly modified in order to have an impact at the Earth centre at the time of the predicted closest approach. The analytical method of Ref. [10] has been used to estimate the deflection, for which the effect of the Earth gravitational interaction is (conservatively) neglected for clarity.

Fig. 4 plots the deflection achievable with a continuous tangential push of magnitude $1 \mathrm{~N}$ applied to both asteroids continuously and starting until 10 years before the predicted impact. In one case (dashed line) a maximum deflection duration of 2 years followed by a coasting phase is assumed while in the other (solid line) the deflection manoeuvre spans up until the predicted impact date.

The 2-years thrust + coasting strategy appears very promising as it is enough to produce a deflection of 2 earth radii and can be carried out with a total spacecraft mass of about $5 \mathrm{t}$ with a $3000 \mathrm{~s}$ specific impulse ion engine (Fig. 2a), or with about $2.2 \mathrm{t}$ should a $10000 \mathrm{~s}$ specific impulse ion
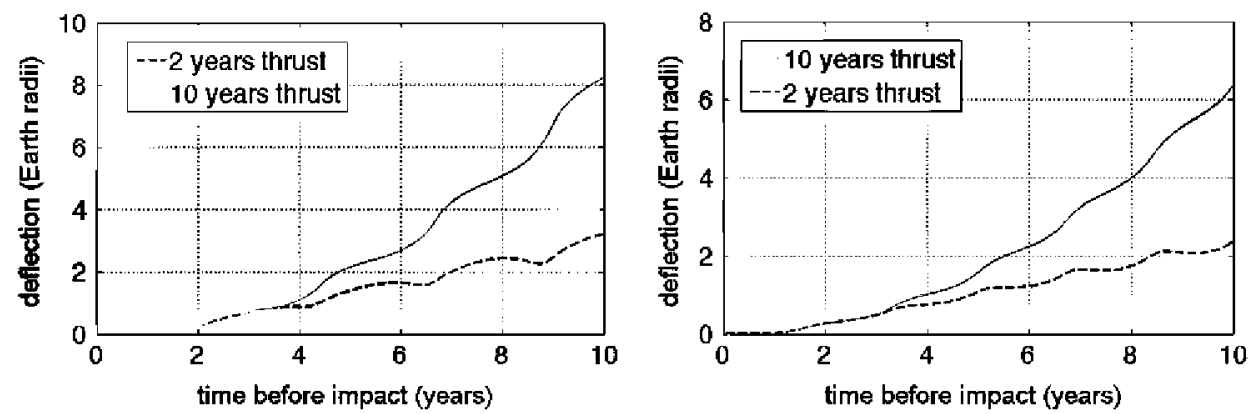

Fig. 4. $b$-plane deflection for constant tangential thrust acceleration ( $F=1 \mathrm{~N}$ ) applied to the asteroid $2007 \mathrm{VK} / 84$ (left) and $2009 \mathrm{AG} 5$ (right) with a warning time of 1-10 years and considering a deflection applied continuously up until expected impact time (solid line) and only for a maximum of 2 years (dashed line). 
engine become available in the near future (Fig. 2b). Note that in order to achieve a $1 \mathrm{~N}$ continuous thrust force a whopping 50-t gravity tractor would be needed (Eq. (7)).

\section{Conclusions}

In this article we have summarised the main characteristics of the recently proposed ion beam shepherd concept (IBS), showing that the method outperforms the gravity tractor methods by more than one order of magnitude for asteroids of less than $200 \mathrm{~m}$ diameter and by a factor of more than five for asteroid smaller than $400 \mathrm{~m}$. The two methods are seen to yield comparable performance only for asteroids of about $2 \mathrm{~km}$ or larger. In particular, the IBS method can be effectively used to deflect a typical $140 \mathrm{~m}$ diameter asteroid with reasonable lead time (10 years from impact) and reasonable spacecraft mass $(<5 \mathrm{t}$ ). Technological advances in the area of ion propulsion and deployable solar arrays as well as guidance and navigation will be the key in order to improve the performance and technological readiness of the concept.

\section{Acknowledgements}

The work was supported by the research project Dynamic simulation of complex space systems supported by the (former) Spanish Ministry of Science and Innovation through Contract AYA2010-18796 and by ESA Situational Awareness ("SSA") Preparatory Programme through project SN-VII NEO impact effects and mitigation measures, Contract No. 4000106175/12/D/MRP.

\section{References}

[1] T. Ahrens, A. Harris, Deflection and fragmentation of near-earth asteroids, Nature 360 (6403) (1992) 429-433.

[2] J.L. Cano, N. Sánchez, M. Sánchez, S. Cornara, Mission analysis for the don quijote phase-a study, in: Proceedings of the 58th Congress of the International Astronautical Federation, Hyderabad, India, September 2007, IAC-07-A3.5.08.

[3] A. Gálvez, l. Carnelli, ESA Studies on the Don Quijote NEO mission: dealing with impact uncertainties, in: Proceedings of the 56th International Astronautical Congress, Fukuoka, Japan, October 2005, Paper IAC-05-Q.P.21.

[4] K. Housen, Collisional fragmentation of rotating bodies, in: Proceedings of the 35th Lunar and Planetary Science Conference, League City, Texas, March 15-19, 2004, Abstract no. 1826.

[5] E. Lu, S. Love, Gravitational tractor for towing asteroids, Nature 438 (7065) (2005) 177-178.

[6] C. Bombardelli, J. Peláez, Ion beam shepherd for asteroid deflection, J Guidance Control Dyn. 34 (4) (2011) 1270-1272.

[7] C. Bombardelli, J. Peláez, lon beam shepherd for contactless debris removal, J. Guidance Control Dyn. 34 (3) (2011) 916-920. May-June.

[8] G. Valsecchi, A. Milani, G. Gronchi, S. Chesley, Resonant returns to close approaches: analytical theory, Astron. Astrophys. 408 (3) (2003) 1179-1196

[9] R. Walker C. Bramanti, O. Sutherland, R. Boswell, C. Charles, D. Fearn, J.G. Del Amo, P.E. Frigot, M. Orlandi, Initial experiments on a dual-stage 4-grid ion thruster for very high specific impulse and power, in: Proceedings of the 42nd AIAA/ASME/SAE/ASEE Joint Propulsion Conference and Exhibit, Washington, DC, AIAA Paper 2006-4669, 2006.

[10] C. Bombardelli, G. Baù, Accurate analytical approximation of asteroid deflection with constant low thrust, in: Celestial Mechanics and Dynamical Astronomy. Online First ${ }^{\mathrm{TM}}, 31$ August 2012. 